\title{
THE NUMBER OF INDEPENDENT SETS IN A GRID GRAPH
}

\author{
NEIL J. CALKIN ${ }^{\dagger}$ AND HERBERT S. WILF $\ddagger$
}

\begin{abstract}
If $f(m, n)$ is the (vertex) independence number of the $m \times n$ grid graph, then we show that the double limit $\eta \stackrel{\text { def }}{=} \lim _{m, n \rightarrow \infty} f(m, n)^{\frac{1}{m n}}$ exists, thereby refining earlier results of Weber [2] and Engel [1]. We establish upper and lower bounds for $\eta$, and prove that $1.503047782 \ldots \leq \eta \leq$ $1.5035148 \ldots$... Numerical computations suggest that the true value of $\eta$ (the "hard square constant") is around 1.5030480824753323. .
\end{abstract}

Key words. independent sets, grid graphs

AMS subject classifications. 05A16, 05C50, 82B20

Let $G_{m, n}$ be the $m \times n$ grid graph. That is, the vertices of $G_{m, n}$ are the $(m+1)(n+1)$ points $(i, j)(0 \leq i \leq m, 0 \leq j \leq n)$ in the plane, and its edges are all of the pairs $(i, j),\left(i^{\prime}, j^{\prime}\right)$ of vertices for which $\left|i-i^{\prime}\right|+\left|j-j^{\prime}\right|=1$. Let $f(m, n)$ be the number of independent sets of vertices in $G_{m, n}$. We study the growth of $f(m, n)$. The figure below shows an independent set $S$ in $G_{4,6}$.

\section{figure= $=$ grid.eps}

Fig. 1

Clearly $G_{m, n}$ contains an independent set of size $\geq m n / 2$, and that set has $\geq 2^{m n / 2}$ subsets, so certainly

$$
\liminf _{m, n \rightarrow \infty} f(m, n)^{\frac{1}{m n}} \geq \sqrt{2}=1.4142 . .
$$

In [2], K. F. E. Weber showed the existence of the limits

$$
\lim _{n \rightarrow \infty} f(m, n)^{1 / m n} \quad \text { and } \quad \lim _{n \rightarrow \infty} f(n, n)^{1 / n^{2}},
$$

and estimated their values. In [1], K. Engel proved some inequalities for these quantities, deduced that $1.50304808 \leq \eta \leq 1.51316067$, and conjectured that $\eta=1.50304808 \cdots$.

We will prove that the double limit

$$
\eta \stackrel{\text { def }}{=} \lim _{m, n \rightarrow \infty} f(m, n)^{\frac{1}{m n}}
$$

exists, and that $1.503047782 \ldots \leq \eta \leq 1.5035148 \ldots$, the latter by exhibiting (relatively) easily computable upper and lower bounds. Numerical computations suggest that the true value of $\eta$ is around 1.5030480824753323.

\section{The transfer matrix}

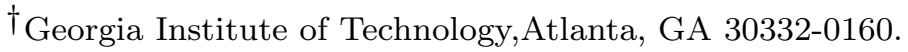

$\ddagger$ University of Pennsylvania, Philadelphia, PA 19104-6395, Supported in part by the Office of Naval Research.
} 
We use the transfer matrix method, in a manner that is similar to the way it was used in [3]. Let $S$ be an independent set in $G_{m, n}$. Consider the portion of $S$ that lies in a fixed column of the graph. This can be regarded as an $(m+1)$-vector of 0's and 1's, in which a 1 indicates that the vertex is in $S$, and a 0 indicates that the vertex is not in $S$. The $(m+1)$-vectors that can arise this way are those that have the property that no two consecutive 1's occur.

Example. Fig. 1 above shows an independent set $S$ in $G_{4,6}$. The portions of $S$ that lie in each of the 7 columns can be represented by the respective 5 -vectors

$$
(0,1,0,1,0),(1,0,0,0,0),(0,0,1,0,1),(0,1,0,1,0),(0,0,1,0,0),(0,1,0,0,0),(1,0,0,1,0) .
$$

Each of these 5-vectors has the property that no two consecutive 1's occur.

Thus, for $m, n$ fixed, we can think of assembling the independent sets of the grid graph by gluing together columns that are chosen from the collection of possible columns, making sure that when we glue an additional column onto the right hand edge of the structure, the new column does not clash with the previous rightmost column.

The collection of possible columns $\mathcal{C}_{m}$ is the set of all $(m+1)$-vectors $\mathbf{v}$, of 0 's and 1's, such that $\mathbf{v}$ contains no two consecutive 1's. The number of these is well known to be $F_{m+2}$, the Fibonacci number.

The condition that vectors $\mathbf{v}^{\prime}, \mathbf{v}^{\prime \prime}$ in $\mathcal{C}_{m}$ are a possible consecutive pair of columns in an independent set of $G_{m, n}$ is simply that they have no 1's in common position, i.e., that $\mathbf{v}^{\prime} \cdot \mathbf{v}^{\prime \prime}=0$ in the sense of the usual dot product of vectors over the reals.

Thus all possible independent sets in the grid graph are obtained by beginning with some vector of $\mathcal{C}_{m}$, and in general, having arrived at some sequence of vectors of $\mathcal{C}_{m}$, adjoin any vector of $\mathcal{C}_{m}$ that is orthogonal to the last one previously chosen, until $(n+1)$ vectors have been selected.

We define a matrix $T=T_{m}$, the transfer matrix of the problem, as follows. $T$ is an $F_{m+2} \times F_{m+2}$ symmetric matrix of 0's and 1's whose rows and columns are indexed by vectors of $\mathcal{C}_{m}$. The entry of $T$ in position $\left(\mathbf{v}^{\prime}, \mathbf{v}^{\prime \prime}\right)$ is 1 if the vectors $\mathbf{v}^{\prime}, \mathbf{v}^{\prime \prime}$ are orthogonal, and is 0 otherwise. $T$ depends only on $m$, not on $n$.

Let $f(m, n, \mathbf{u})$ denote the number of independent sets of $G_{m, n}$ whose rightmost column vector is $\mathbf{u}$. Then clearly we have

$$
f(m, n+1, \mathbf{v})=\sum_{\mathbf{u} \in \mathcal{C}_{m}} f(m, n, \mathbf{u}) T_{\mathbf{u}, \mathbf{v}} \quad\left(n \geq 0 ; \mathbf{v} \in \mathcal{C}_{m}\right)
$$

or, in matrix-vector notation, $\mathbf{f}_{n+1}=T \mathbf{f}_{n}$, with $\mathbf{f}_{0}=\mathbf{1}$, the vector whose entries are all 1's. It follows that $\mathbf{f}_{n}=T^{n} \mathbf{1}$, for all $n \geq 0$. The number of independent sets of $G_{m, n}$ is the sum of the entries of the vector $\mathbf{f}_{n}$. Thus

$$
f(m, n)=\mathbf{1} \cdot T^{n} \mathbf{1}
$$

i.e., $f(m, n)$ is the sum of all of the entries of the matrix $T^{n}$. 
Since $T$ has nonnegative entries, its dominant eigenvector cannot be orthogonal to $\mathbf{1}$, and so we have at once that $\lim _{n \rightarrow \infty} f(m, n)^{1 / n}$ exists for each $m$, and is equal to $\Lambda_{m}$, the largest eigenvalue of the (real, symmetric) transfer matrix $T$ (the existence of that limit also follows from an obvious subadditivity argument). It follows that

$$
\liminf \Lambda_{m}^{1 / m}=\liminf _{m, n} f(m, n)^{1 / m n} \leq \limsup _{m, n} f(m, n)^{1 / m n}=\lim \sup \Lambda_{m}^{1 / m}
$$

We remark in passing that some interesting generating functions can be found in moderately explicit form. Indeed, since $f(m, n)$ is the sum of the entries of $T^{n}$, we see that for $m$ fixed, the numbers $f(m, n)$ can be read off as the coefficient of $x^{n}$ in the power series expansion of the sum of the entries of the matrix $(I-x T)^{-1}$.

For instance, take $m=2$. The possible column vectors in an independent set are

$$
\text { (000), (001), (010), (100), (101). }
$$

If we index the rows and columns in this order, then the transfer matrix is

$$
T=\left(\begin{array}{lllll}
1 & 1 & 1 & 1 & 1 \\
1 & 0 & 1 & 1 & 0 \\
1 & 1 & 0 & 1 & 1 \\
1 & 1 & 1 & 0 & 0 \\
1 & 0 & 1 & 0 & 0
\end{array}\right)
$$

If we find the sum of the entries of $(I-x T)^{-1}$ we see that $f(2, n)$ is the coefficient of $x^{n}$ in

$$
\frac{5+7 x-x^{2}-x^{3}}{1-2 x-6 x^{2}+x^{4}}=5+17 x+63 x^{2}+227 x^{3}+827 x^{4}+2999 x^{5}+10897 x^{6}+O\left(x^{7}\right) .
$$

\section{The lower bound}

In this section we will first prove the existence of the limit $\eta$, and then by a slight refinement of the argument, we will give a close lower bound for $\eta$.

By the maximum principle, since the transfer matrix $T$ is real and symmetric, we have, for every positive integer $p$,

$$
\Lambda_{m}^{p} \geq \frac{\left(\mathbf{1}, T_{m}^{p} \mathbf{1}\right)}{(\mathbf{1}, \mathbf{1})}
$$

where we now explicitly exhibit the dependence of the transfer matrix on $m$ by the subscript. But $\left(\mathbf{1}, T_{m}^{p} \mathbf{1}\right)=\left(\mathbf{1}, T_{p}^{m} \mathbf{1}\right)$, since both sides count the independent sets in the grid graph $G_{m, p}$. Thus, after taking $m$ th roots we have

$$
\left(\Lambda_{m}^{1 / m}\right)^{p} \geq\left(\frac{\left(\mathbf{1}, T_{p}^{m} \mathbf{1}\right)}{(\mathbf{1}, \mathbf{1})}\right)^{1 / m} .
$$


Now take the liminf of both sides of this inequality, as $m \rightarrow \infty$. We obtain

$$
\left(\liminf _{m \rightarrow \infty} \Lambda_{m}^{1 / m}\right)^{p} \geq \frac{\Lambda_{p}}{\frac{1+\sqrt{5}}{2}}
$$

Now take the $p$ th root, and the $\lim \sup$ as $p \rightarrow \infty$ to discover that

$$
\liminf _{m \rightarrow \infty} \Lambda_{m}^{1 / m} \geq \limsup _{p \rightarrow \infty} \Lambda_{p}^{1 / p}
$$

The reverse inequality being obvious, we have that the $\operatorname{limit}_{\lim } \rightarrow \infty \Lambda_{m}^{1 / m}$ exists, and hence by (3) so does the limit

$$
\eta=\lim _{m, n} f(m, n)^{1 / m n}=\lim _{m \rightarrow \infty} \Lambda_{m}^{1 / m}
$$

Next we will refine the above argument to obtain a good numerical lower bound for $\eta$. We replace (4) by

$$
\Lambda_{m}^{p} \geq \frac{\left(T_{m}^{q} \mathbf{1}, T_{m}^{p} T_{m}^{q} \mathbf{1}\right)}{\left(T_{m}^{q} \mathbf{1}, T_{m}^{q} \mathbf{1}\right)}
$$

which by the maximum principle is true for every positive integer $q$. But the right side can be rewritten as

$$
\frac{\left(\mathbf{1}, T_{m}^{q} T_{m}^{p} T_{m}^{q} \mathbf{1}\right)}{\left(\mathbf{1}, T_{m}^{q} T_{m}^{q} \mathbf{1}\right)}=\frac{\left(\mathbf{1}, T_{m}^{p+2 q} \mathbf{1}\right)}{\left(\mathbf{1}, T_{m}^{2 q} \mathbf{1}\right)}=\frac{\left(\mathbf{1}, T_{p+2 q}^{m} \mathbf{1}\right)}{\left(\mathbf{1}, T_{2 q}^{m} \mathbf{1}\right)}
$$

where we have again used the fact that $\forall p, m: T_{m}^{p}=T_{p}^{m}$. Hence

$$
\eta^{p}=\lim _{m \rightarrow \infty}\left(\Lambda_{m}^{1 / m}\right)^{p} \geq \frac{\Lambda_{p+2 q}}{\Lambda_{2 q}}
$$

and so

$$
\eta \geq\left(\frac{\Lambda_{p+2 q}}{\Lambda_{2 q}}\right)^{\frac{1}{p}}
$$

\section{Example.}

We now work out the case $p=2, q=1$ of this lower bound. The transfer matrix $T_{2}$ is shown in (2) above, and its largest eigenvalue is the largest zero of $1-6 x^{2}-2 x^{3}+x^{4}=0$, i.e., $\Lambda_{2}=3.6313812604036 \ldots$... 
The transfer matrix $T_{4}$ is $13 \times 13$, and it is given by

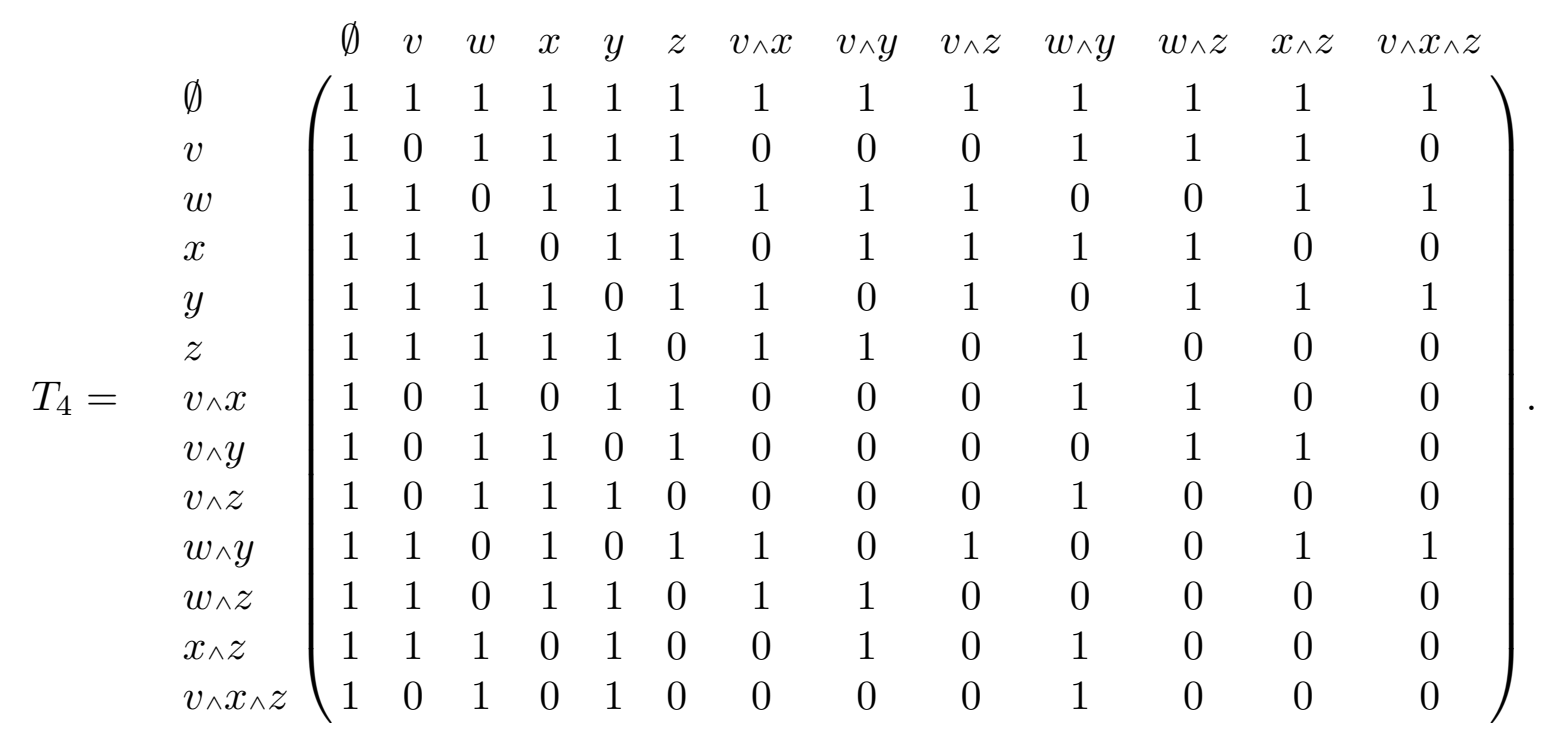

The largest eigenvalue of $T_{4}$ is the largest root of the equation

$$
1-4 t-20 t^{2}+64 t^{3}+15 t^{4}-105 t^{5}+36 t^{6}+4 t^{7}-t^{8}=0,
$$

namely $\Lambda_{4}=8.2032591937550246879103 \ldots$. Hence we have the lower bound

$$
\eta \geq\left(\frac{\Lambda_{4}}{\Lambda_{2}}\right)^{\frac{1}{2}}=1.502994159 \ldots
$$

We have in fact worked out the case $p=2, q=3$, though we will not show the details here, with the result that $\eta \geq 1.503047782 \ldots$..

\section{The upper bound}

In this section we will exhibit another transfer matrix problem with the property that it provides upper bounds for the problem in which we are interested. Further, the upper bounding problem will be independent of $m, n$, and will depend on a new integer parameter $p$. There will be a valid upper bound for each positive integer $p$.

For each positive integer $p$, the largest eigenvalue of the transfer matrix $T$ obviously satisfies

$$
\Lambda_{m} \leq \operatorname{Trace}\left(T^{2 p}\right)^{1 / 2 p}
$$

and indeed the right side approaches the left for $p \rightarrow \infty$. But

$$
\operatorname{Trace}\left(T^{2 p}\right)=\sum T_{x_{0}, x_{1}} T_{x_{1}, x_{2}} \cdots T_{x_{2 p-1}, x_{0}}
$$

Now each term in this sum is 0 or 1 , hence the sum is equal to the number of good $2 p$-tuples of subsets of $1,2, \ldots, m$, that is, the number of $2 p$-tuples $\left(x_{0}, x_{1}, \ldots, x_{2 p-1}\right)$ of subsets of $1,2, \ldots, m$ for which 
(a) each $x_{i}$ contains no two consecutive entries, and

(b) each consecutive (on the circle) pair of $x$ 's is disjoint.

We will find another way to count these tuples that will enable us to eliminate the dependence on $m$ completely.

Define the associated transfer matrix $B_{2 p}$ to be the matrix whose rows and columns are indexed by all subsets of $2 p$ abstract "objects" which contain no two objects that are consecutive (on the circle). The matrix is $N_{p} \times N_{p}$, where $N_{p}=F_{2 p-1}+F_{2 p+1}$, and the $F$ 's are the Fibonacci numbers. The entries of this matrix are

$$
\left(B_{2 p}\right)_{X, Y}= \begin{cases}1, & \text { if } X \cap Y=\emptyset ; \\ 0, & \text { otherwise. }\end{cases}
$$

Note that this matrix is independent of $m$. Its utility rests in the following fact.

Proposition. The trace of $T^{2 p}$ is equal to the sum of all of the entries of the matrix $B_{2 p}^{m-1}$.

Proof. Consider a nonvanishing term of $\operatorname{Trace}\left(T^{2 p}\right)$, say the term $T_{x_{0}, x_{1}} T_{x_{1}, x_{2}} \cdots T_{x_{2 p-1}, x_{0}}$. Now each $x_{i}$ is a subset of $1,2, \ldots, m$. Define sets

$$
S_{j}=\left\{k:(0 \leq k \leq 2 p-1) \wedge\left(j \in x_{k}\right)\right\} \quad(j=1,2, \ldots, m) .
$$

Then each $S_{j}$ is a subset of $2 p$ objects. Each $S_{j}$ contains no two objects that are consecutive on the circle for otherwise $j$ would belong to two consecutive $x_{k}$ 's on the circle. Further $S_{i}, S_{i+1}$ (on the circle) are disjoint, for otherwise two consecutive letters $i, i+1$ would both belong to one of the $x_{k}$ 's, a contradiction.

Hence this collection of sets $S_{j}$ corresponds to a nonvanishing expression

$$
\left(B_{2 p}\right)_{S_{1}, S_{2}}\left(B_{2 p}\right)_{S_{2}, S_{3}} \cdots\left(B_{2 p}\right)_{S_{m-1}, S_{m}} .
$$

But this is just one of the terms in the expansion of the sum of all of the entries of the matrix $B_{2 p}^{m-1}$, i.e., in the expansion of $\left(\mathbf{1}, B_{2 p}^{m-1} \mathbf{1}\right)$.

Conversely, consider a nonvanishing term of $\left(\mathbf{1}, B_{2 p}^{m-1} \mathbf{1}\right)$, say the term shown in (9) above. Define a $(2 p)$-tuple $\left(x_{0}, \ldots, x_{2 p-1}\right)$ of subsets of $1,2, \ldots, m$ by

$$
x_{j}=\left\{i:(1 \leq i \leq m) \wedge\left(j \in S_{i}\right)\right\} \quad(j=0,1, \ldots, 2 p-1) .
$$

Then each $x_{i}$ has no two consecutive entries, for otherwise $j$ would belong to two consecutive $S_{i}$ 's and one of the factors $\left(B_{2 p}\right)_{S_{i}, S_{i+1}}$ would vanish. Likewise, each consecutive (on the circle) pair of sets $x_{j}$ is disjoint, for otherwise some $S_{i}$ would contain two consecutive (on the circle) values of $j$. This completes the proof of the proposition.

Now for each fixed positive integer $p$ we have

$$
\Lambda_{m} \leq \operatorname{Trace}\left(T^{2 p}\right)^{1 / 2 p}=\left(\mathbf{1}, B_{2 p}^{m-1} \mathbf{1}\right) .
$$

If we take the $m$ th root and then the limit as $m \rightarrow \infty$, we find that

$$
\eta=\lim _{m, n \rightarrow \infty} f(m, n)^{1 / m n}=\limsup _{m \rightarrow \infty} \Lambda_{m}^{1 / m} l e \xi_{2 p}^{1 / 2 p},
$$


where $\xi_{2 p}$ is the largest eigenvalue of $B_{2 p}$.

\section{Example.}

We consider the case $2 p=6$.

Here, Trace $\left(T^{6}\right)$ is the number of good 6-tuples $(u, v, w, x, y, z)$, such that each of the six is a subset of $[1, m]$, none of them contains any two consecutive elements, and all of the pairs

$$
(u, v),(v, w),(w, x),(x, y),(y, z),(z, u)
$$

are disjoint pairs. Thus, a single letter $i$ might belong to any of the 18 combinations

$$
\emptyset, u, v, w, x, y, z, u_{\wedge} w, u_{\wedge} x, u \wedge y, v \wedge x, v \wedge y, v \wedge z, w \wedge y, w \wedge z, x_{\wedge} z, u_{\wedge} w \wedge y, v_{\wedge} x_{\wedge} z .
$$

The associated transfer matrix $B_{6}$ is $18 \times 18$, and its entries are 1 or 0 depending on whether the membership combination that is labeled by the row is disjoint from the membership combination that is labeled by the column. The full matrix $B_{6}$, with its lines labelled in the order shown above, is

$$
\left(\begin{array}{llllllllllllllllll}
1 & 1 & 1 & 1 & 1 & 1 & 1 & 1 & 1 & 1 & 1 & 1 & 1 & 1 & 1 & 1 & 1 & 1 \\
1 & 0 & 1 & 1 & 1 & 1 & 1 & 1 & 0 & 0 & 1 & 1 & 1 & 1 & 1 & 1 & 0 & 1 \\
1 & 1 & 0 & 1 & 1 & 1 & 1 & 1 & 1 & 1 & 0 & 0 & 0 & 1 & 1 & 1 & 1 & 0 \\
1 & 1 & 1 & 0 & 1 & 1 & 1 & 0 & 1 & 1 & 1 & 1 & 1 & 0 & 0 & 1 & 0 & 1 \\
1 & 1 & 1 & 1 & 0 & 1 & 1 & 1 & 0 & 1 & 0 & 1 & 1 & 1 & 1 & 0 & 1 & 0 \\
1 & 1 & 1 & 1 & 1 & 0 & 1 & 1 & 1 & 0 & 1 & 0 & 1 & 0 & 1 & 1 & 0 & 1 \\
1 & 1 & 1 & 1 & 1 & 1 & 0 & 1 & 1 & 1 & 1 & 1 & 0 & 1 & 0 & 0 & 1 & 0 \\
1 & 0 & 1 & 0 & 1 & 1 & 1 & 0 & 0 & 0 & 1 & 1 & 1 & 0 & 0 & 1 & 0 & 1 \\
1 & 0 & 1 & 1 & 0 & 1 & 1 & 0 & 0 & 0 & 0 & 1 & 1 & 1 & 1 & 0 & 0 & 0 \\
1 & 0 & 1 & 1 & 1 & 0 & 1 & 0 & 0 & 0 & 1 & 0 & 1 & 0 & 1 & 1 & 0 & 1 \\
1 & 1 & 0 & 1 & 0 & 1 & 1 & 1 & 0 & 1 & 0 & 0 & 0 & 1 & 1 & 1 & 1 & 0 \\
1 & 1 & 0 & 1 & 1 & 0 & 1 & 1 & 1 & 0 & 0 & 0 & 0 & 0 & 1 & 1 & 0 & 0 \\
1 & 1 & 0 & 1 & 1 & 1 & 0 & 1 & 1 & 1 & 0 & 0 & 0 & 1 & 0 & 0 & 1 & 0 \\
1 & 1 & 1 & 0 & 1 & 0 & 1 & 0 & 1 & 0 & 1 & 0 & 1 & 0 & 0 & 1 & 0 & 1 \\
1 & 1 & 1 & 0 & 1 & 1 & 0 & 0 & 1 & 1 & 1 & 1 & 0 & 0 & 0 & 0 & 0 & 0 \\
1 & 1 & 1 & 1 & 0 & 1 & 0 & 1 & 0 & 1 & 0 & 1 & 0 & 1 & 0 & 0 & 1 & 0 \\
1 & 0 & 1 & 0 & 1 & 0 & 1 & 0 & 0 & 0 & 1 & 0 & 1 & 0 & 0 & 1 & 0 & 1 \\
1 & 1 & 0 & 1 & 0 & 1 & 0 & 1 & 1 & 1 & 0 & 0 & 0 & 1 & 0 & 0 & 1 & 0
\end{array}\right)
$$

Calculation then reveals that the largest eigenvalue of $B_{6}$ is the largest root of the equation

$$
-1+2 t+25 t^{2}+3 t^{3}-12 t^{4}+t^{5}=0
$$

namely $11.55170956604814509 \ldots$... Therefore

$$
\eta=\lim _{m, n} f(m, n)^{1 / m n} \leq 11.55170956604814509 \ldots{ }^{1 / 6}=1.503514809475903023 \ldots
$$

\section{References}


1. Konrad Engel, On the Fibonacci number of an $m \times n$ lattice, Fibonacci Quart. 28 (1990), 72-78.

2. Karl Weber, On the number of stable sets in an $m \times n$ lattice. Rostock. Math. Kolloq. 34 (1988), 28-36. (MR 89i:05172)

3. Herbert S. Wilf, The problem of the kings, Electronic J. Combinatorics 2 (1995), \#R3. 